\title{
Spectral Problems of Two-Parameter System of Operators
}

\author{
Rakhshanda Dzhabarzadeh \\ Department of functional analysis, Institute of Mathematics and Mechanics of NAS of Azerbaijan, Baku, Azerbaijan
}

Email address:

rakhshanda.dzhabarzade@rambler.ru

\section{To cite this article:}

Rakhshanda Dzhabarzadeh. Spectral Problems of Two-Parameter System of Operators. Pure and Applied Mathematics Journal. Special Issue: Spectral Theory of Multiparameter Operator Pencils and Its Applications. Vol. 4, No. 4-1, 2015, pp. 33-37. doi: $10.11648 /$ j.pamj.s.2015040401.17

\begin{abstract}
The author has proved the existence of the multiple basis of the eigen and associated vectors of the two parameter system of operators in Hilbert spaces. The proof essentially uses the theorem of the existence of multiple basis of operator bundles and the notion of the abstract analog of resultant of two operator pencils, acting, generally speaking, in different Hilbert spaces. Considerable non-selfadjoint two parameter systems depend on both parameters in a complicated manner.
\end{abstract}

Keywords: Multiparameter, Spectrum, Operator, Space, Eigenvector

\section{Introduction}

Spectral theory of operators is one of the important directions of functional analysis. The method of separation of variables in many cases turned out to be the only acceptable, since it reduces finding a solution of a complex equation with many variables to finding a solution of a system of ordinary differential equations, which are much easier to study.

F.V. Atkinson [1] studied the fragmentary results for multiparameter symmetric differential systems, built multiparameter spectral theory of selfadjoint systems in finite-dimensional Euclidean spaces. Further, by taking the limit, Atkinson generalized the results obtained for the multiparameter systems with self-adjoint operators in finite dimensional space on the case of the multiparameter system with compact selfadjoint operators in Hilbert spaces.
Later Browne, Sleeman, Roch and other mathematicians built the spectral theory of selfadjoint multiparameter system in infinite-dimensional Hilbert spaces [2],[3].

In this work the existence of multiple basis on eigen and associated vectors of two parameter non-selfadjoint system of operators in Hilbert spaces is proved. Definitions of the associated vectors, multiple completeness of eigen and associated vectors of two-parameter non-selfadjoint systems, are introduced in [4],[6].

\section{Preliminary Definitions and Remarks}

Let

$$
\begin{aligned}
& A(\lambda, \mu)=A_{0}+\lambda A_{1}+\cdots+\lambda^{m_{1}} A_{m_{1}}+\mu A_{m_{1}+1}+\cdots+\mu^{n_{1}} A_{m_{1}+n_{1}}+\sum_{r<m_{1}, s<n_{1}} \lambda^{r} \mu^{s} A_{r, s} \\
& B(\lambda, \mu)=B_{0}+\lambda B_{1}+\cdots+\lambda^{m_{21}} B_{m_{2}}+\mu B_{m_{2}+1}+\cdots+\mu^{n_{2}} B_{m_{2}+n_{2}}+\sum_{r<m_{2}, s<n_{2}} \lambda^{r} \mu^{s} B_{r, s}
\end{aligned}
$$

be the non-linear multiparameter system in two parameters.

Operators $A_{i}$ (correspondingly, $B_{i}$ ) act in the Hilbert space $H_{1}$ (correspondingly, $H_{2}$ ), $H_{1} \otimes H_{2}$ is a tensor product of spaces $H_{1}$ and $H_{2}$.

For nonlinear algebraic system with two variables sufficient conditions of existence of solutions are given. The proof of these statements are received as a corollary of more common reviewing considered in this paper.

Definition 1.

$(\lambda, \mu)$ is an eigenvalue of the system (1) depending on two spectral parameters if there are such non-zero pair vectors $x \in H_{1}, y \in H_{2}$ that the equations (2) 


$$
\begin{aligned}
& A(\lambda, \mu) x=\left(A_{0}+\lambda A_{1}+\cdots+\lambda^{m_{1}} A_{m_{1}}+\mu A_{m_{1}+1}+\cdots+\mu^{n_{1}} A_{m_{1}+n_{1}}+\sum_{r<m_{1}, s<n_{1}} \lambda^{r} \mu^{s} A_{r, s}\right) x=0 \\
& B(\lambda, \mu) y=\left(B_{0}+\lambda B_{1}+\ldots+\lambda^{m_{2}} B_{m_{2}}+\mu B_{m_{2}+1}+\ldots+\mu_{n_{2}} B_{m_{2}+n_{2}}+\sum \lambda^{r} \mu^{s} B_{r, s}\right) y=0
\end{aligned}
$$

are satisfied .Decomposable element $x \otimes y$ is called an eigen vector of multiparameter system (1).

Definition 2 [1]

Operator $A_{i}^{+}$(correspondingly, $B_{i}^{+}$) is induced into the space $H=H_{1} \otimes H_{2}$ by the operator $A_{i}$ (correspondingly $B_{i}$ ), acting in the space $H_{1}$ (correspondingly, $H_{2}$ ), if the following conditions satisfy: on decomposable tensor $x \otimes y$,
$A_{i}^{+}(x \otimes y)=\left(A_{i} x\right) \otimes y$ and,$B_{i}^{+}(x \otimes y)=x \otimes\left(B_{i} y\right)$, on other elements of the space $H=H_{1} \otimes H_{2}$-on linearity and continuity

Definition 3 ([4], [5])

A tensor $z_{m_{1}, m_{2}}$ is named $\left(m_{1}, m_{2}\right)-$ th the associated vector to an eigenvector $z_{0,0}=x \otimes y$, if the following conditions (3) are satisfied

$$
\sum_{0 \leq r_{i} \leq k_{i}} \frac{1}{r_{1} ! r_{2} !} \frac{\partial^{r_{1}+r_{2}} A^{+}(\lambda, \mathcal{E})}{\partial^{r_{1}} \lambda \partial^{r_{2}}} z_{k_{1}-r_{1}, k_{2}-r_{2}}=0, \sum_{0 \leq r_{i} \leq k_{i}} \frac{1}{r_{1} ! r_{2} !} \frac{\partial^{r_{1}+r_{2}} B^{+}(\lambda, \varepsilon)}{\partial^{r_{1}} \lambda \partial^{r_{2}}} z_{k_{1}-r_{1}, k_{2}-r_{2}}=0, k_{s} \leq m_{s} ; i=1,2 ; s=1,2
$$

$\left(k_{1}, k_{2}\right)$ - arrangements from set of the whole nonnegative numbers on 2 with possible recurring and zero.

Under canonical system of e.a.vectors (this definition is generalization of the definition of canonical system, introduced in [10] in the case polynomial pencils in one parameter) for $(\lambda, \mu)$ we understand the system

$$
\left\{z_{i_{1}, i_{2}}^{(k)}\right\}_{0 \leq i_{r} \leq m_{r}}, r=1,2
$$

possessing the following properties: elements $z_{0,0}^{(k)}$ form basis of a eigen subspace $M\left(\lambda^{0}\right)$; there is $z_{0,0}^{(1)}$ eigenvector which multiplicity reaches a possible maxima $p_{1}+1$; there is $z_{0,0}^{(k)}$ eigenvector which is not expressing linearly through $z_{0,0}^{(1)}, \ldots$, $z_{0,0}^{(k-1)}$ which sum of multiplicities reaches a possible maxima $p_{k}+1$ Let's designate through $M\left(\left(\lambda^{0}, \mu^{0}\right)\right)$ a subspace spanned by eigen and associated vectors of the system (1), corresponding to an eigenvalue $\lambda^{0}$.

Linearly-independent elements form a chain of a set $\left(z_{i_{1}, i}\right) \subset H$ eigen and associated (e.a) vectors of (1). The multiplicity of eigenvalue designates the greatest number of associated vectors to $z_{0, \ldots, 0}$ a plus 1 .

The sum $p_{1}+p_{2}+\ldots+p_{s}+s$ is a multiplicity of an eigen value $\left(\lambda^{0}, \mu^{0}\right)$.

Elements in (4) form a chain of eigen and associated vectors for every fixed value $k, k=1,2, \ldots, s$.

\section{Definition 4.}

Systems of elements $\left\{x_{k, 1}\right\}_{1}^{r}(k=1,2, \ldots, n)$ of Hilbert space form multiple bases in this space if any $n$ elements $f_{1}, f_{2}, \ldots, f_{n}$ of space can be spread out in series $f_{i}=\sum_{k=1}^{s} c_{i} x_{k, i}(i=1, \ldots, n)$ with coefficients, not dependent on an index of vectors $f_{1}, f_{2}, \ldots, f_{n}$. If system $\left\{x_{k, 1}\right\}_{k=1}^{r}$ coincides with the system of eigen and associated vectors of an operator, and systems $\left\{x_{k, i}\right\}_{k=1}^{r}$ are constructed, proceeding from sequences on $\left\{x_{k, 1}\right\}_{k=1}^{r}$ according to some rules then speak about $n$-multiple basis on system of eigen and associated vectors of an operator

Definition5( [6],[7])

Let be two operator pencils depending on the same parameter and acting in, generally speaking, various Hilbert spaces

$$
\begin{aligned}
& A(\lambda)=A_{0}+\lambda A_{1}+\lambda^{2} A_{2}+\ldots+\lambda^{n} A_{n} \\
& B(\lambda)=B_{0}+\lambda B_{1}+\lambda^{2} B_{2}+\ldots+\lambda^{m} B_{m}
\end{aligned}
$$

Operator $\operatorname{Re} s(A(\lambda), B(\lambda))$ is presented by the matrix

$$
\left(\begin{array}{cccccc}
A_{0} \otimes E_{2} & A_{1} \otimes E_{2} & \ldots & A_{n} \otimes E_{2} & \ldots & 0 \\
\cdot & \cdot & \ldots & \cdot & \ldots & \cdot \\
0 & 0 & \ldots A_{0} \otimes E_{2} & A_{1} \otimes E_{2} & \ldots & A_{n} \otimes E_{2} \\
E_{1} \otimes B_{0} & E_{1} \otimes B_{1} & \ldots & E_{1} \otimes B_{m} & . . & 0 \\
. & \cdot & \ldots & . & \ldots & . \\
. & . & \ldots E_{1} \otimes B_{0} & E_{1} \otimes B_{1} & \ldots . & E_{1} \otimes B_{m}
\end{array}\right)
$$

which acts in the $\left(H_{1} \otimes H_{2}\right)^{n+m}$ - direct sum of $n+m$ copies of the space $H_{1} \otimes H_{2}$. In a matrix (5) number of rows with operators $A_{i}$ is equal to leading degree of the parameter $\lambda$ in pencils $B(\lambda)$ and the number of rows with $B_{i}$ is equal to the leading degree of parameter $\lambda$ in $A(\lambda)$. Notion of abstract analog of resultant of two operator pencils is considered in the [8] for the case of the same leading degree of the parameter in both pencils and in the [7] for, generally speaking, different leading degrees of the parameters in the operator pencils.

Theorem 1.

Let all the operators be bounded in corresponding Hilbert spaces, one of operators $A_{n}$ or $B_{m}$ has bounded inverse Then operator pencils $A(\lambda)$ and $B(\lambda)$ have a common point of spectra if and only if 


$$
\operatorname{Ker} \operatorname{Re} s(A(\lambda), B(\lambda)) \neq\{\vartheta\}
$$

Remark 1. If the Hilbert spaces $H_{1}$ and $H_{2}$ are the finite dimensional spaces then a common points of spectra of operator pencils $A(\lambda)$ and $B(\lambda)$ are their common eigenvalues.(see [6], [7].)

\section{Multiple Basis of Eigen and Associated Vectors of Multiparameter System with Two Parameters}

Consider the system (2). Operators $A_{i}, B_{i}$ act in the Hilbert spaces $H_{1}$ and $H_{2}$,correspondingly . For study of the spectral properties of the system (1) we use the notion of abstract analog of resultant of $A(\lambda)$ and $B(\lambda)$. Fix the one of the parameters in (1). Let it is the parameter $\lambda$ and $\lambda=\lambda_{0}$. Then we have two operator pencils in one parameter $\mu$.

$$
\begin{aligned}
& A\left(\lambda_{0}, \mu\right) x=\left(A_{0}+\lambda_{0} A_{1}+\cdots+\lambda_{0}^{m_{1}} A_{m_{1}}+\mu A_{m_{1}+1}+\cdots+\mu^{n_{1}} A_{m_{1}+n_{1}}+\sum_{r<m_{1}, s<n_{1}} \lambda_{0}^{r} \mu^{s} A_{r, s}\right) x=0 \\
& B\left(\lambda_{0}, \mu\right) y=\left(B_{0}+\lambda_{0} B_{1}+\cdots+\lambda_{0}^{m_{21}} B_{m_{2}}+\mu B_{m_{2}+1}+\cdots+\mu^{n_{2}} B_{m_{2}+n_{2}}+\sum_{r<m_{2}, s<n_{2}} \lambda_{0}^{r} \mu^{s} B_{r, s}\right) y=0
\end{aligned}
$$

Arrange the pencils on increasing of the degree of the parameter $\mu$ and denote the operator coefficients of the parameter $\mu$ in the degree $s$ in the operator pencil $A(\lambda, \mu)$ though $A_{s}+\sum_{r<m_{1}} \lambda^{r} A_{r, s}=\tilde{A}_{s}(\lambda)$ and in the pencil $B(\lambda, \mu)$ operator coefficient of the parameter $\mu$ in degree $s$ we

$$
\tilde{A}\left(\lambda_{0}\right)=A_{0}+\lambda_{0} A_{1}+\cdots+\lambda_{0}{ }^{m_{1}} A_{m_{1}}, \tilde{B}\left(\lambda_{0}\right)=B_{0}+\lambda_{0} B_{1}+\cdots+\lambda_{0}{ }^{m_{1}} B_{m_{1}}
$$

Construct the resultant of operator pencils $A\left(\lambda_{0}, \mu\right)$ and $B\left(\lambda_{0}, \mu\right)$ (the parameter $\lambda$ is fixed).

$$
\operatorname{Re} s\left(A\left(\lambda_{0}, \mu\right), B\left(\lambda_{0}, \mu\right)\right)=\left(\begin{array}{ccccccc}
\tilde{A}^{+}\left(\lambda_{0}\right) & \tilde{A}_{m_{1}+1}^{+}\left(\lambda_{0}\right) & \ldots & \tilde{A}_{m_{1}+n_{1}}^{+} & 0 & \ldots & 0 \\
0 & \tilde{A}^{+}\left(\lambda_{0}\right) & \ldots & \tilde{A}_{m_{1}+n_{1}-1}^{+}\left(\lambda_{0}\right) & \tilde{A}_{m_{1}+n_{1}}^{+} & \ldots & 0 \\
\cdot & \cdot & . & \cdot & \cdot & \ldots & . \\
0 & 0 & \ldots \tilde{A}^{+}\left(\lambda_{0}\right) & \tilde{A}_{m_{1}+1}^{+}\left(\lambda_{0}\right) & \tilde{A}_{m_{1}+2}^{+}\left(\lambda_{0}\right) & \ldots & \tilde{A}_{m_{1}+n_{1}}^{+} \\
\tilde{B}^{+}\left(\lambda_{0}\right) & \tilde{B}_{m_{2}+1}^{+}\left(\lambda_{0}\right) & \ldots & \tilde{B}_{m_{2}+n_{2}} & 0 & \ldots & 0 \\
0 & \tilde{B}^{+}\left(\lambda_{0}\right) & \ldots & \tilde{B}_{m_{2}+n_{2}-1}^{+}\left(\lambda_{0}\right) & \tilde{B}_{m_{2}+n}^{+} & \ldots & 0 \\
\cdot & \cdot & \ldots & \cdot & \cdot & \ldots & . \\
0 & 0 & \ldots \tilde{B}\left(\lambda_{0}\right)^{+} & \tilde{B}_{m_{2}+1}^{+}\left(\lambda_{0}\right) & \tilde{B}_{m_{2}+2}^{+}\left(\lambda_{0}\right) & \ldots & \tilde{B}_{m_{2}+n_{2}}^{+}
\end{array}\right)
$$

The number of rows with the operators $\tilde{A}_{s}(\lambda)$ is equal to the leading degree of the parameter $\mu$ in the operator pencil $B\left(\lambda_{0}, \mu\right)$, that is $n_{1}$; number of rows with the operators $\tilde{B}_{s}^{+}(\lambda)$ is equal to the leading degree of the parameter $\mu$ in the pencil $A(\lambda, \mu)$, that is $n_{1}$.

$$
\text { Let } \quad \max _{0 \leq i \leq n_{1}}\left(k_{i} n_{2}+i m_{2}\right)=k_{j} n_{2}+j m_{2} \quad \text { realizes at }
$$

$i=j, 0 \leq j \leq n_{1}$ where $i=s, 0 \leq s \leq n_{2}$. Let the greatest degree of $\lambda_{0}$ in the operator coefficient of $\mu^{s}\left(s=m_{1}+1, \ldots, m_{1}+n_{1}-1\right)$ in the operator pencil $A\left(\lambda_{0}, \mu\right)$ be $k_{s}$. By analogy the greatest degree of $\lambda_{0}$ in the operator coefficients at $\mu^{s}, s=m_{2}+1, \ldots, m_{2}+n_{2}-1$ in the operator pencil $B\left(\lambda_{0}, \mu\right)$ be $r_{s}$. So the parameter $\lambda_{0}$ is fixed arbitrarily, further, in the system we miss the index 0 of the parameter $\lambda_{0}$.

Let $K$ denotes the term of the expansion of the resultant (7) free from parameter $\lambda$ and let $K$ be a selfadjoint operator with the $\operatorname{ker} K=\{\vartheta\}$.

It is known that the self-adjoint completely continuous operator has a discrete spectrum, i.e. the spectrum of the operator contains only eigenvalues of finite multiplicity. If the series of modules of eigenvalues of completely continuous operator in some positive degree converges then this operator belongs to class $\sigma_{p}$.

Arrange the bundle produced by the decomposition of the resultant (7), to increase the power of the parameter $\lambda$ and denote the operator coefficients of $\lambda^{s}$ though $T_{i}^{+}$.

Let's remind, that under an eigen value of polynomial bundle $\quad L(\lambda)=A_{0}+\lambda A_{1} B+\ldots+\lambda^{n-1} A_{n-1} B^{n-1}+\lambda^{n} B^{n} \quad$ is 
understood such non-zero vector $x_{0}$, that equality $L\left(\lambda_{0}\right) x_{0}=x_{0}$ is satisfied.. $m$-th associated vector $x_{k}$ to an eigenvector $x_{0}$ is the vector, satisfying to conditions $x_{k}=L\left(\lambda_{0}\right) x_{k}+\frac{d}{d \lambda} L\left(\lambda_{0}\right) x_{k-1}+\ldots+\frac{1}{k !} \frac{d^{k}}{d \lambda^{k}} L\left(\lambda_{0}\right) x_{0} ; k=1, \ldots, m$

Let $\left\{x_{k, 0}\right\}$ be system of eigen and associated vectors of a bundle $L(\lambda)$.

On system of eigen and associated vectors of polynomial bundle $L(\lambda)$ derivative systems of vectors are constructed by rules

$$
x_{i, j}=\left\{\frac{d^{j}}{d t^{j}} e^{\lambda_{0} t}\left(x_{i, 0}+x_{i-1,0} \frac{t}{1 !}+\ldots+x_{0,0} \frac{t^{i}}{i !}\right\}_{t=0}, i=1, \ldots\right.
$$

\section{Spectral Properties of Two Parameter System}

For the study of the spectral problems of two parameter system (1) we use the following result

In a separable Hilbert space we consider an operator pencil

$$
L(\lambda)=A_{0}+\lambda A_{1} B+\lambda^{2} A_{2} B^{2}+\ldots+\lambda^{n-1} A_{n-1} B^{n-1}+\lambda^{n} B^{n}
$$

when the operators $A_{i}, B$ are completely continuous in the space $H, \lambda \in C$

Let $B(\operatorname{Ker} B=\{\vartheta\})$ be the normal operator, characteristic values of which lie on a finite number of rays, emanating from the origin. Denote an increasing sequence of modules characteristic numbers of the operator $B$, taking into account their multiplicity.

Theorem 2.[5] Suppose that one of the following two conditions:

a)

$$
0<p<1, \underset{k \rightarrow \infty}{\lim _{k \rightarrow \infty}} k \mu_{k}^{-p}=0
$$

operators

$A_{i} B^{-p}(i=0,1,2, \ldots, n-1)$ are bounded

b)

$$
0<p<1, \lim _{k \rightarrow \infty} k \mu_{k}^{-p}<\infty, \quad A_{i} B^{-p}(i=0,1,2, \ldots, n-1)
$$

completely continuous operators are satisfied.

Then different eigenvalues $\left\{\lambda_{n}\right\}_{1}^{\infty}$ of $L(\lambda)$ can be arranged in a sequence such that for some increasing sequence $\left\{n_{j}\right\}_{1}^{\infty}\left(n_{0}=0\right)$ of positive integers, and for all $n$ elements $f_{0}, f_{1}, \ldots, f_{n-1}$ of the space $H$, satisfying the conditions $f_{i} \in R\left(B^{i}\right)$, the expansions

$$
f_{j}=\sum_{i=0}^{\infty}\left(\sum_{k=n_{i-1}}^{n_{i}-1} \sum_{s=0}^{m_{k_{s}}-1} \sum_{r=0}^{p_{s_{k}}-1} a_{s r}^{(k)} Z_{j s r}^{(k)}\right)
$$

where $p_{s_{k}}$ the number of different eigenvectors of multiplicity $m_{k r}$ corresponding to eigenvalue $\lambda_{k}$ with eigenvector $L(\lambda)$

$$
Z_{j s r}^{(k)}=\left[\frac{d^{j}}{d t^{j}} e^{\lambda_{k} t}\left(Z_{0 s r}^{(k)}+Z_{0, s-1, r}^{(k)} \frac{t}{1 !}+\ldots+Z_{00 r} \frac{t^{s}}{s !}\right)\right]_{t=0}
$$

$\left\{Z_{0 s k}^{(k)}\right\}$-canonical system, corresponding eigen values $\lambda_{k}$ are satisfied. .

Arrange the bundle produced by the decomposition of the resultant (8), to increase the power of the parameter $\lambda$ and denote the operator coefficients of $\lambda^{s}$ though $T_{s}^{+}$and the modules of characteristic numbers of operators $S, S_{1}, S_{2}$ though $\mu_{S}, \mu_{s_{1}}, \mu_{s_{2}}$, correspondingly. $S, S_{1}, S_{2}$ are defined below.

Theorem3. Let be

$$
k_{j} n_{2}+j m_{2}>r_{s} n_{1}+s m_{1}, \quad S=K^{-\frac{1}{2}} A_{k_{j}, j}^{n_{2}} \otimes B_{m_{2}}^{s} K^{-\frac{1}{2}} \in \sigma_{p} \quad \text { is }
$$

selfadjoint completely continuous opertator and one of following conditions are fulfilled

a) $0<p<1, \underline{\lim }_{k \rightarrow \infty} k\left(\mu_{s}\right)_{k}^{-p}=0$ operators $\left(T_{i}^{\bullet}\right) S^{-\frac{i p}{k_{j} n_{2}+j m_{2}}}$, $i=1, \ldots, k_{j} n_{2}+j m_{2} \operatorname{Ker} S=\{\vartheta\}$ are bounded,

$$
\text { b) } 0<p<1, \underset{k \rightarrow \infty}{\lim _{k \rightarrow \infty}} k\left(\mu_{s}\right)_{k}^{-p}<0 \quad \text { operators }\left(T_{i}^{\bullet}\right) S^{-\frac{i p}{k_{j} n_{2}+j m_{2}}}
$$

are the completely continuous

Then characteristic values $\left\{\mu_{n}\right\}_{1}^{\infty}$ of $\operatorname{Re} s(A(\mu(\lambda)), B(\mu(\lambda))$ can be arranged in a sequence such that for some increasing sequence $\left\{n_{j}\right\}_{1}^{\infty}\left(n_{0}=0\right)$ of positive integers, and for all $n$ elements $f_{0}, f_{1}, \ldots, f_{n-1}$ of the space $H$, satisfying the conditions $f_{i} \in R\left(S^{i}\right)$, we have the expansions

$$
f_{j}=\sum_{i=0}^{\infty}\left(\sum_{k=n_{i-1}}^{n_{i}-1} \sum_{s=0}^{m_{k_{s}}-1} \sum_{r=0}^{p_{s_{k}}-1} a_{s r}^{(k)} Z_{j s r}^{(k)}\right)
$$

where $p_{s_{k}}$ the number of different eigenvectors of multiplicity $m_{k r}$ corresponding to eigenvalue $\lambda_{k}=\frac{1}{\mu_{k}}$.

Proof of the Theorem3.

If any of the conditions a), b) is satisfied then conditions of Theorem 2 are fulfilled, therefore, multiple basis of eigen and associated vectors of the operator bundle $\operatorname{Re} s(A(\lambda, \mu), B(\lambda, \mu))$ exists. Studies conducted in the papers [4],[5] and [6] show that the eigenvectors of the bundle (8)(decompositions of resultant) are either eigenvectors or associated vectors of the system (1) in the definition of which there is no differentiation on $\lambda$. The associated vectors of the bundle (8) (decomposition of the resultant (7)) are also eigenvectors of the system (1). The results of the theorem1 mean if the conditions a), b) of the theorem 2 hold operator pencils in (1) have the common point of spectra (in the finite dimensional Hilbert space $H_{1} \otimes H_{2}$ the common point of spectra is the common eigenvalues ). Really, each condition a), b) of the theorem 2 means the $\operatorname{ker} \operatorname{Re} s(A(\lambda, \mu), B(\lambda, \mu)) \neq 0$. Consider the decomposition of the resultant (8) which is the operator 
pencil in the parameter $\lambda$. Consequently, eigen and associated vectors of this operator pencil form the multiple basis in the tensor product space $H_{1} \otimes H_{2}$ and the multiplicity of this basis coincides with the greatest degree of the parameter $\lambda$ in the operator pencil $\operatorname{Re} s(A(\lambda, \mu), B(\lambda, \mu)$ ) (the parameter $\lambda$ is fixed) . Earlier in the [4],[5],[6] it is proved that the system of eigen and associated vectors of obtained pencil, depending on parameter $\lambda$ and coincides with the system of eigen and associated vectors of the system (1).Therefore, the eigen and associated vectors of the two parameter system (1) form the $\operatorname{Max}\left(k_{j} n_{2}+j m_{2}, r_{s} n_{1}+s m_{1}\right)$ multiple basis in $H_{1} \otimes H_{2}$. Thus, the systems of eigen and associated vectors of (1) and the resulting expansion of the resultant $\operatorname{Re} s(A(\lambda, \mu), B(\lambda, \mu))$ simultaneously $\operatorname{Max}\left(k_{j} n_{2}+j m_{2}, r_{s} n_{1}+s m_{1}\right)$ multiple basis with brackets.

Theorem 4

Let be $r_{s} n_{1}+s m_{1}>k_{j} n_{2}+j m_{2}$, operator $S_{1}=K^{-\frac{1}{2}} A_{m_{1}}^{s} \otimes B_{r_{j}, s}^{n_{1}} K^{-\frac{1}{2}} \in \sigma_{p} \quad$ has $\quad$ inverse, $\quad$ selfadjoint completely continuous and one of following conditions

a) $0<p<1, \underline{\lim }_{k \rightarrow \infty} k\left(\mu_{s_{1}}\right)_{k}^{-p}=0$

$\tilde{T}_{i} S_{1}^{-\frac{i p}{r_{s} n_{1}+s m_{1}}}\left(i=1,2, \ldots, r_{s} n_{1}+s m_{1}\right)$ are bounded operators

b) $0<p<1, \underline{\lim }_{k \rightarrow \infty} k\left(\mu_{s_{1}}\right)_{k}^{-p}<0$

$\tilde{T}_{i} S_{1}^{-\frac{i p}{r_{s} n_{1}+s m_{1}}}\left(i=1,2, \ldots, r_{s} n_{1}+s m_{1}\right)$ are completely complete operators

is satisfied. Then the assertions of theorem 3 is fulfilled.

Theorem 5. Let be $r_{s} n_{1}+s m_{1}=k_{j} n_{2}+j m_{2}$, operator $S_{2}=K^{-\frac{1}{2}}\left(A_{k_{j}, j}^{n_{2}} \otimes B_{m_{2}}^{s}+(-1)^{n_{1} n_{2}} A_{m_{1}}^{j} \otimes B_{r_{s}, s}^{n_{1}}\right) K^{-\frac{1}{2}} \in \sigma_{p} \quad$ has inverse, selfadjoint completely continuous and one of following conditions

$$
\begin{aligned}
& \left.c_{1}\right)^{0<p<1,} \underline{\lim }_{k \rightarrow \infty} k\left(\mu_{s_{1}}\right)_{k}^{-p}=0 \\
& \tilde{T}_{i} S_{2}^{-\frac{i p}{r_{s} n_{1}+s m_{1}}}\left(i=1,2, \ldots, r_{s} n_{1}+s m_{1}\right) \text { are bounded } \\
& \left.c_{2}\right)^{0<p<1, ~} \underset{k \rightarrow \infty}{\lim } k\left(\mu_{s_{1}}\right)_{k}^{-p}=0
\end{aligned}
$$$$
\tilde{T}_{i} S_{2}^{-\frac{i p}{r_{s} n_{1}+s m_{1}}} \quad\left(i=1,2, \ldots, r_{s} n_{1}+s m_{1}\right) \quad \text { completely continuous }
$$

operators is satisfied. Then the statements of the theorem 3 is fulfilled.

The next theorem is the special case of the theorem2

Theorem6. Let all operators $A_{i}$ (correspondingly, $B_{i}$ ) act in finite dimensional Hilbert space $H_{1}$ (correspondingly, $H_{2}$ ), and one of following conditions:

$$
\begin{aligned}
& \text { a) } \quad k_{j} n_{2}+j m_{2}>r_{s} n_{1}+s m_{1}, \quad \operatorname{Ker}\left(A_{k_{j}, j}^{n_{2}} \otimes B_{m_{2}}^{s}\right)=\{\vartheta\} \quad, \\
& A_{k_{j}, j}^{\bullet}=A_{k_{j}, j} ; B_{m_{2}}=B_{b_{2}}^{\cdot}
\end{aligned}
$$

b) $r_{s} n_{1}+s m_{1}>k_{j} n_{2}+j m_{2}$, operator $A_{m_{1}}^{s} \otimes B_{r_{s}, s}^{n_{1}}$ has inverse and selfadjoint

c) $r_{s} n_{1}+s m_{1}=k_{j} n_{2}+j m_{2}$,operator $\quad A_{k_{j, j}}^{n_{2}} \otimes B_{m_{2}}^{s}+$ $+(-1)^{n_{1} n_{2}} A_{m_{1}}^{j} \otimes B_{r_{s}, s}^{n_{1}}$ has inverse and selfadjoint

is satisfied. Then the eigen and associated vectors of the system (2) form $\operatorname{Max}\left(k_{j} n_{2}+j m_{2}, r_{s} n_{1}+s m_{1}\right)$ multiple basis in the tensor product of the space $H_{1} \otimes H_{2}$.

Remark1. Eigen and associated vectors are the first components of the elements of the kernel of the resultant of operator pencils $A(\lambda, \mu)$ and $B(\lambda, \mu)$.

Remark2. If the $H_{1} \otimes H_{2}$ is infinite dimensional Hilbert space then the system of eigen and associated vectors of the system (1) coincides with the system of eigen and associated vectors of the operator pencil obtaining as result of decomposition of the resultant of operators

Theorems 4,5,6 are proved by analogy with the proof of the Theorem 3.

\section{Conclusion}

In this paper it is given the conditions of multiple basis of eigen and associated vectors of two parameter system of operators in Hilbert space $H_{1} \otimes H_{2}$.

\section{References}

[1] Atkinson F.V. Multiparameter spectral theory. Bull.Amer.Math.Soc.1968, 74, 1-27.

[2] Browne P.J. Multiparameter spectral theory. Indiana Univ. Math. J,24, 3, 1974

[3] Sleeman B.D. Multiparameter spectral theory in Hilbert space. Pitnam Press, London, 1978, p.118.

[4] Dzhabarzadeh R.M. Spectral theory of two parameter system in finite-dimensional space. Transactions of NAS Azerbaijan, v. 3-4 1998, p.12-18

[5] Dzhabarzadeh R.M. About expansion on eigen and associated vectors of operator pencil polynomially depending on parameters. Scientific notes of Azerbaijan State University 1964, № 3,c.75-81

[6] Dzhabarzadeh R.M.Spectral theory of multiparameter system of operators in Hilbert space, Transactions of NAS of Azerbaijan, 1-2, 1999, 33-41

[7] Balinskii A.I Generation of notions of Bezutiant and Resultant DAN of Ukr. SSR, ser.ph.-math and tech. of sciences, 1980,2. (in Russian).

[8] Khayniq (Хайниг Г). Abstract analog of Resultant for two polynomial bundles Functional analyses and its applications, 1977, 2 , no. 3, p.94-95

[9] Dzhabarzadeh R.M . On solutions of nonlinear algebraic systems with two variables. Pure and Applied Mathematics, Journal, vol. 2, No. 1, pp. 31-37, 2013

[10] Keldish M .V. On completeness of eigen functions of some classes of linear nonselfadjoint operators .Successes of Mathematical Sciences (УMH), 1971, v.27, issue.4, pp..15-47 (in Russian) 